\title{
A New Model to Study Healing of a Complex Femur Fracture with Concurrent Soft Tissue Injury in Sheep
}

\author{
Martin E. Wullschleger ${ }^{1 *}$, Roland Steck ${ }^{1 * \#}$, Romano Matthys $^{2}$, John D. Webster ${ }^{1}$, \\ Maria A. Woodruff ${ }^{1}$, Devakar R. Epari ${ }^{1}$, Keita Ito $^{3}$, Michael A. Schuetz ${ }^{1,4}$ \\ ${ }^{1}$ Institute of Health and Biomedical Innovation, Queensland University of Technology, Brisbane, Australia; ${ }^{2}$ AO Research Institute, \\ Davos, Switzerland; ${ }^{3}$ Department of Biomedical Engineering, Eindhoven University of Technology, Eindhoven, Netherlands; \\ ${ }^{4}$ Trauma Service, Princess Alexandra Hospital, Brisbane, Australia. \\ Email: "r.steck@qut.edu.au
}

Received April 6 ${ }^{\text {th }}, 2013$; revised May $8^{\text {th }}, 2013$; accepted May 19 ${ }^{\text {th }}, 2013$

Copyright (C) 2013 Martin E. Wullschleger et al. This is an open access article distributed under the Creative Commons Attribution License, which permits unrestricted use, distribution, and reproduction in any medium, provided the original work is properly cited.

\begin{abstract}
High energy bone fractures resulting from impact trauma are often accompanied by subcutaneous soft tissue injuries, even if the skin remains intact. There is evidence that such closed soft tissue injuries affect the healing of bone fractures, and vice versa. Despite this knowledge, most impact trauma studies in animals have focussed on bone fractures or soft tissue trauma in isolation. However, given the simultaneous impact on both tissues a better understanding of the interaction between these two injuries is necessary to optimise clinical treatment. The aim of this study was therefore to develop a new experimental model and characterise, for the first time, the healing of a complex fracture with concurrent closed soft tissue trauma in sheep. A pendulum impact device was designed to deliver a defined and standardised impact to the distal thigh of sheep, causing a reproducible contusion injury to the subcutaneous soft tissues. In a subsequent procedure, a reproducible femoral butterfly fracture (AO C3-type) was created at the sheep's femur, which was initially stabilised for 5 days by an external fixator construct to allow for soft tissue swelling to recede, and ultimately in a bridging construct using locking plates. The combined injuries were applied to twelve sheep and the healing observed for four or eight weeks (six animals per group) until sacrifice. The pendulum impact led to a moderate to severe circumferential soft tissue injury with significant bruising, haematomas and partial muscle disruptions. Posttraumatic measurements showed elevated intra-compartmental pressure and circulatory tissue breakdown markers, with recovery to normal, pre-injury values within four days. Clinically, no neurovascular deficiencies were observed. Bi-weekly radiological analysis of the healing fractures showed progressive callus healing over time, with the average number of callus bridges increasing from 0.4 at two weeks to 4.2 at eight weeks. Biomechanical testing after sacrifice showed increasing torsional stiffness between four and eight weeks healing time from $10 \%$ to $100 \%$, and increasing ultimate torsional strength from 10\% to $64 \%$ (relative to the contralateral control limb). Our results demonstrate the robust healing of a complex femur fracture in the presence of a severe soft tissue contusion injury in sheep and demonstrate the establishment of a clinically relevant experimental model, for research aimed at improving the treatment of bone fractures accompanied by closed soft tissue injuries.
\end{abstract}

Keywords: Fracture; Ovine; Large Animal Model; Bone; Soft Tissue Injury

\section{Introduction}

High energy bone fractures resulting from impact trauma, such as those sustained by pedestrians hit by a motor vehicle, are often accompanied by subcutaneous soft tissue injuries, even if the skin remains intact. Clinical experience and experimental research has provided evidence that the healing of bone fractures is influenced by the state of the surrounding tissue [1-3]. The muscles and

\footnotetext{
*These authors contributed equally to this study.
}

\#Corresponding author. periosteum that surround bone are not only important for the re-vascularisation of the fracture callus, but these tissues are also known as a source for osteoprogenitor cells that are essential for bone formation during fracture healing [3-5]. A better understanding of the interplay between bone fracture healing and the surrounding soft tissue is therefore essential for the development of better treatment modalities for bone fractures with concurrent soft tissue injuries. Conducting animal experiments that closely represent a clinical scenario is challenging, espe- 
cially in large animals. The experimental trauma should reflect a clinically observed injury pattern, whilst at the same time allow for a high level of standardisation, which is required to limit the variability and therefore reduce the necessary sample size.

While numerous experimental models can be found in the literature to assess either soft tissue or bone fracture healing individually, there are only a few studies that have investigated the interaction between these two injuries. Furthermore, most of these studies are conducted in small animal models. Although small animal experimental models are highly valuable to address fundamental research questions pertaining to the interaction between biochemical processes occurring during bone fracture and soft tissue trauma healing, it can be argued that large animal models are best suited to addressing immediate clinical research questions.

To our knowledge, the only experiment in larger animals combining a soft tissue injury and a bone fracture has been reported by Schmeling and colleagues, who studied a moderate soft tissue injury and a concurrent simple tibia shaft fracture in sheep [6]. In humans, high energy trauma to the tibia (which has very limited soft tissue coverage) commonly results in open fractures. And while open tibia fractures are clinically demanding and often lead to complications due to wound contamination and blood and tissue loss, it is also the closed fractures with extensive subcutaneous soft tissue trauma that are particularly challenging to treat. Specifically, due to the inaccessibility of the damaged soft tissue structures and the absence of any quantitative clinical measures to assess the closed soft tissue injuries objectively, the diagnosis and monitoring of closed soft tissue trauma is difficult. We therefore believe that the model by Schmeling et al is not optimal for replicating the most challenging injury scenarios with regard to the injury location and the fracture type. In addition, while Schmeling's experiments and most other fracture healing experiments have been performed on simple osteotomies or transverse fractures, these injuries are not reflective of the multifragmentary fracture patterns typically observed as a result of high energy impact trauma with extensive soft tissue trauma. Consequently, more realistic and reproducible trauma experimental models are required, in order to develop and assess methods, fixation implants and procedures that may improve the clinical treatment of combined closed soft tissue and bone injuries.

The goal of this project was therefore to develop an experimental model with a standardised closed soft tissue injury of grade III, according to the Tscherne classification [7], and a reproducible, co-localised multi-frag-mentary fracture type $\mathrm{C} 3$, according to the AO classification [8], in a clinically relevant and challenging anatomical region, such as the distal femur. In this commu- nication, we report on the characterisation of the healing of the combined injuries in this experimental sheep model.

\section{Materials and Methods}

\subsection{Injury Model}

Based on pilot experiments and a review of the literature, the application of the injuries was divided into two successive procedures with the initial application of a soft tissue injury followed by the creation of a multi-fragmentary fracture of the sheep femur.

All procedures were carried out with approval from the University Animal Ethics Committee (UAEC) of the Queensland University of Technology (Ref No: 4222A), Brisbane, Australia and followed the policies and guidelines of the NHMRC and AVCC (including the Australian Code of Practice for the care and use of animals for scientific purposes (7th Edition 2004).

The combined soft tissue trauma and multi-fragmentary fracture was applied to 12 Merino wethers (mean age: $5.6 \pm 0.9$ years and mean weight: $40.0 \pm 5.6 \mathrm{~kg}$ ). Induction of anaesthesia was achieved with propofol (10 $\mathrm{mg} / \mathrm{ml}) 0.5 \mathrm{mg} / \mathrm{kg}$ as bolus intravenous injection and maintained at $4 \mathrm{mg} / \mathrm{kg} /$ hour. Buprenorphine $(0.01 \mathrm{mg} / \mathrm{kg})$ was administered intravenously to provide analgesia.

\subsection{Application of Soft Tissue Trauma}

A pendulum impact device (Figure 1(a)) was developed to create a standardised soft tissue trauma with a defined injury pattern that is comparable to that resulting from a high energy impact in humans. The anaesthetised sheep was placed onto a trestle, with the right femur precisely positioned with the anvils at the location of the subsequent impact, five centimetres proximal from the knee joint. The impactor and counterpart anvils were designed to compress the entire circumference of the distal region of the thigh, while sparing the bone (Figure 1(b)). In terminal in vivo experiments, the soft tissue injury resulting from the impact was characterised by post-mortem macroscopic dissections confirming the severe damage with subcutaneous haematomas and layer disruption, intramuscular haematomas as well as partial muscle disruption.

\subsection{Application of Multi-Fragmentary Fracture}

After application of the soft tissue trauma, a multi-fragmentary shaft fracture (AO C-type fracture) was created on the distal femur through a small $(3 \mathrm{~cm})$ antero-lateral, trans-muscular approach. The configuration of this fracture was defined by two transverse fracture lines approx $3 \mathrm{~cm}$ apart, and a longitudinal fracture line dividing the central, tubular bone section into two pieces, resulting in 


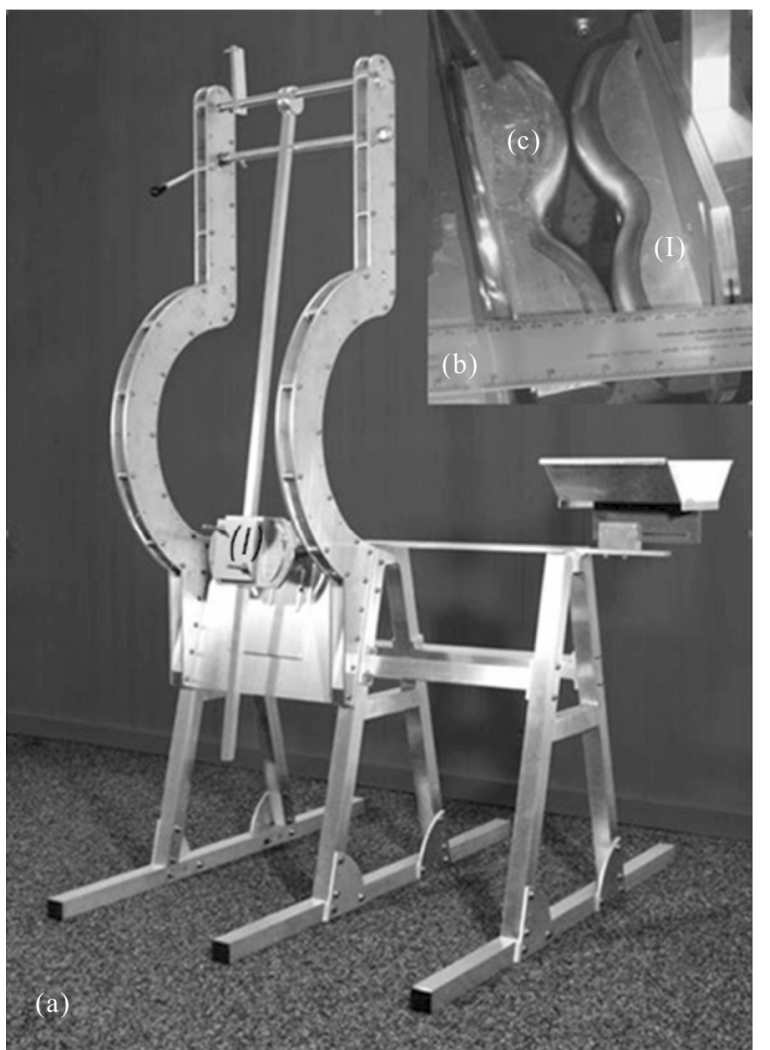

Figure 1. (a) Pendulum trestle to create the soft tissue injury. The sheep is placed onto the trestle and the right femur positioned between the impactor and counterpart; (b) The impactor (I) and counterpart (C) are shaped to compress the entire circumference of the thigh muscles, while sparing the bone.

four main fragments (Figure 2). The detailed steps to create this injury are illustrated in Figure 3.

After application of the soft tissue trauma and the multifragmentary fracture, the distal femur fracture was temporarily stabilised with a lateral external fixator in a bridging construct, consisting of two bicortical Schanz' screws in the distal and proximal main fragments, connected by two carbon rods (Large External Fixator, Synthes, Switzerland). The two central, smaller fragments were manually reduced but not stabilised. Five days after trauma and initial stabilisation, consistent with clinical practice to allow for soft tissue swelling to recede, the external fixator was removed and definitive fixation of the fracture was achieved with an internal fixator (7-hole narrow 4.5/5.0 mm LCP, Synthes, Switzerland) with two bicortical locking screws on either side of the fracture zone.

\subsection{Characterisation of Fracture Healing}

Immediately post operatively, conventional $\mathrm{x}$-rays were taken in anterior-posterior (a-p) and latero-medial (1-m) planes to confirm the fracture pattern and the successful
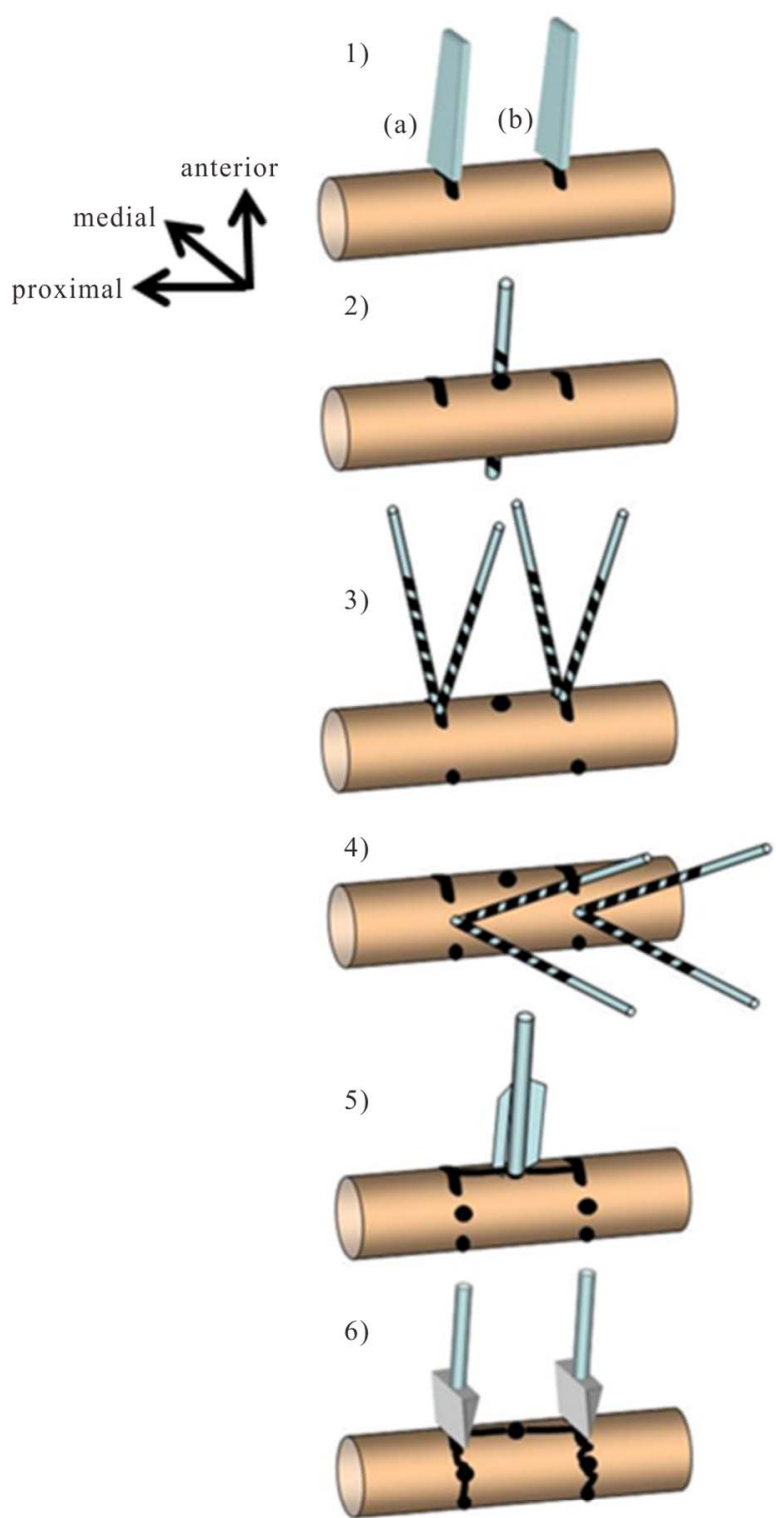

Figure 2. Fracture creation sequence: 1) Two partial transverse anterior osteotomies are performed, $30 \mathrm{~mm}$ apart from each other; 2) In the middle between the osteotomies one antero-posterior (a-p), bicortical drill hole is placed; 3) Through the anterior osteotomies, four bicortical, oblique holes are drilled at 30 degrees to each other; 4) In the latero-medial direction: four bicortical holes are drilled at 30 degrees to each other; 5) To split the middle segment longitudinally into two fragments, a blade bar is inserted into the a-p drill hole with gentle hits by a hammer; 6) Finally, two chisels are used to initiate the two transverse fractures by a strong hit with the hammer.

reduction (Figure 4). Further bi-planar x-rays were taken fortnightly to monitor the fracture healing progress until the animals were sacrificed at 4 weeks $(n=6)$ and 8 weeks $(n=6)$ (Figure 4).

The digitised radiographs were evaluated qualitatively, with respect to fracture configuration (fracture pattern 

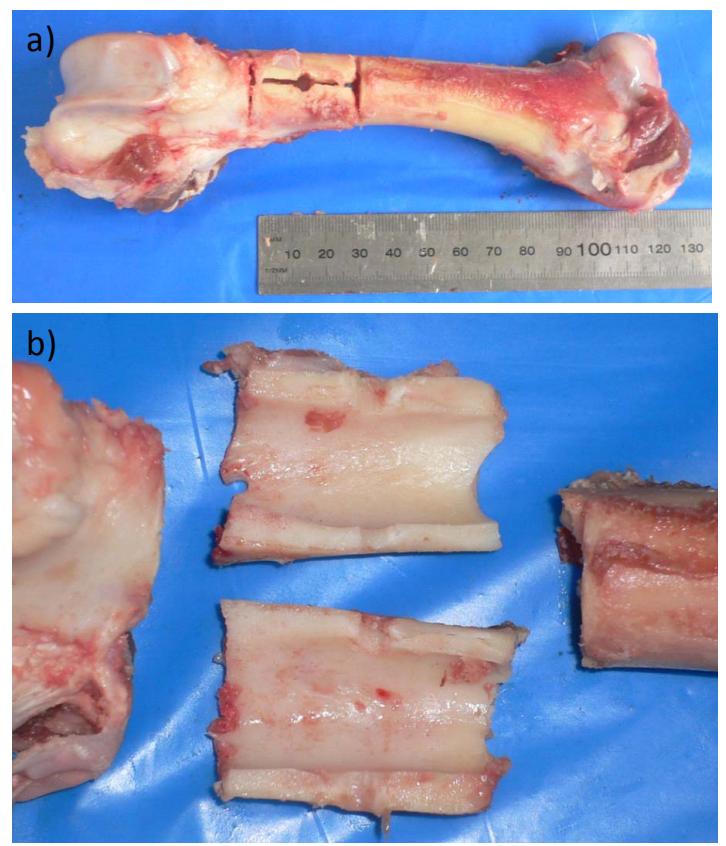

Figure 3. (a) A cadaveric ovine femur during fracture creation in vitro (AO-type C3 fracture). Two partial transverse osteotomies, one longitudinal osteotomy and the drill holes have been applied; (b) The fracture initiation with chisels and a so-called blade bar results in four major fragments with this fracture model.

according to the AO classification) and quantitatively with the computer software AMIRA (Visage Imaging $\mathrm{GmbH}$, Berlin, Germany) to determine the reproducibility of the fracture pattern. This was defined by the fracture length as an average value from six measurements: The length of the fracture zone and the distance between the proximal and distal main fragment was measured in three different aspects in radiographs from two planes. In addition, the progress of fracture healing was assessed by counting the number of callus bridges across the entire fracture zone. Of the 12 experimental animals, six animals were sacrificed after four weeks and another six animals at eight weeks after trauma. Both femora were harvested, and following removal of the plate fixator and dissection of all but the intact muscles, the proximal and distal bone ends were embedded in dental acrylic PMMA (Paladur, Heraeus Kulzer, Germany). The femora were tested in torsion on an Instron mechanical testing machine (Instron 8874, Instron Pty Ltd., Norwood, Massachusetts, USA). The maximum torque at failure and torsional stiffness of the healing femora was determined and reported as a percentage of the values from the intact contra-lateral side.

\subsection{Monitoring Soft Tissue Healing}

In order to characterise the soft tissue damage and its recovery over time, an intramuscular probe (Neurovent PTO, Raumedic AG, Germany) was inserted percutaneA-P view

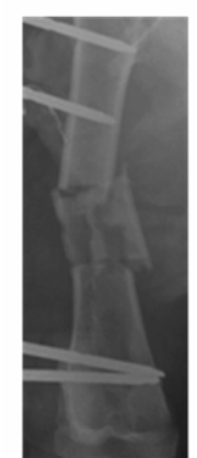

postop 1

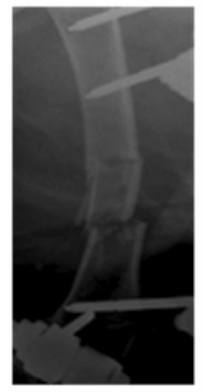

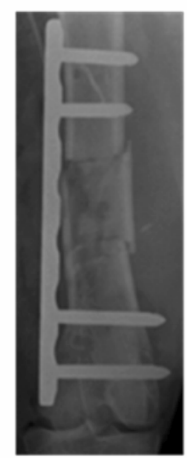

postop 2

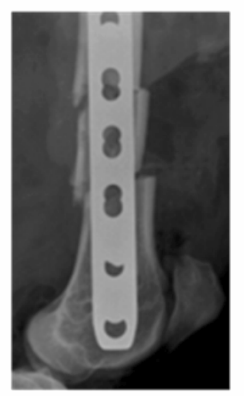

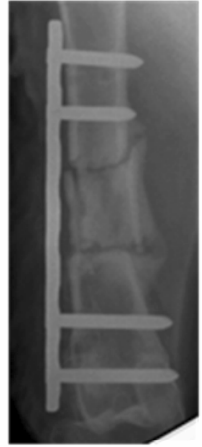

4 weeks

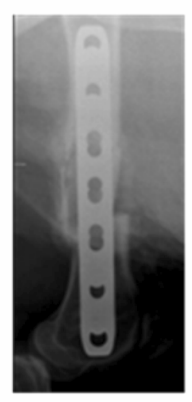

Lateral view

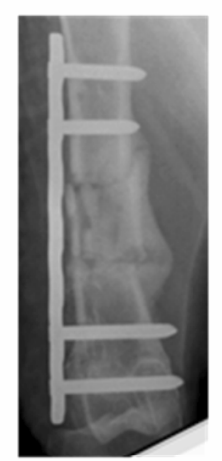

6 weeks

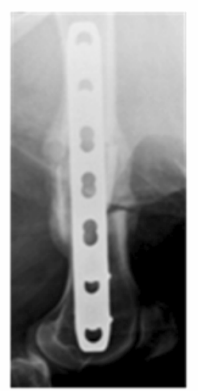

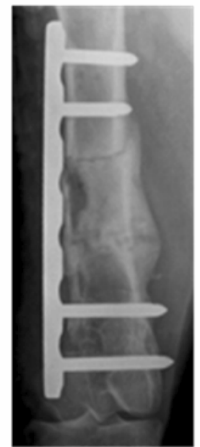

8 weeks

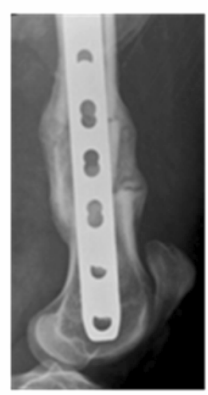

Figure 4. Representative $x$-ray series of the fracture healing progress. 
ously, immediately after application of the soft tissue trauma: The sensor was positioned in the distal part of the rectus femoris muscle of the anterior muscle compartment at the level of the soft tissue damage and the fracture site, which was confirmed by manual palpation through a small incision. In 7 animals the intra-compartmental pressure could be measured twice daily over a period of 5 days post surgery. Venous blood samples were taken daily from one day preoperatively to 5 days postoperatively from all 12 animals to determine the systemic levels of serum markers for muscle tissue breakdown, Creatine Kinase (CK) and Lactate Dehydrogenase (LDH).

\subsection{Statistical Analysis}

Statistical analysis was performed using SPSS software version 17 (SPSS Inc., Chicago IL, USA). All data were tested for normal distribution with the KolmogorovSmirnov test and parametric testing was performed using ANOVA. All numerical data are reported as mean values \pm standard deviation and were considered significant at $\mathrm{p}<$ 0.05 .

\section{Results}

\subsection{Characterisation of Fracture Healing}

A multi-fragmentary fracture, classified as AO-C3 fracture according to $\mathrm{AO}$ classification, with an average length of the fracture zone of $30.1 \pm 3.1 \mathrm{~mm}$ was created in 12 sheep.

All fractures were stabilised with the same external fixator construct initially, and after a complication-free period of five days to allow soft tissue recovery, the fractures were definitively stabilised with an internal fixator plate.

In the analysis of the $x$-ray images an increasing average number of callus bridges of $0.4 \pm 0.5,0.9 \pm 0.8,2.5 \pm$ 0.6 and $3.2 \pm 0.8$ at two, four, six and eight weeks, respectively, demonstrated progressive fracture healing $(\mathrm{p}$ $=0.08$ between 2 and 4 weeks, $\mathrm{p}<0.05$ between 4 and 6 weeks, $\mathrm{p}=0.11$ between 6 and 8 weeks). This observation was confirmed by the mechanical testing results after 4 and 8 weeks (Figure 5). The torsional rigidity of the fractured femur significantly increased from $9.8 \% \pm$ $12.4 \%(n=6)$ at 4 weeks to $100.4 \% \pm 41.4 \%(n=6)$ at 8 weeks ( $\mathrm{p}<0.005)$. Similarly, the ultimate torque improved from $9.6 \% \pm 9.9 \%$ to $64.4 \% \pm 18.3 \%$ ( $<<0.005)$.

\subsection{Monitoring of Soft Tissue Injury}

The intra-compartmental pressure measurements in the quadriceps muscle of 7 animals revealed peak values immediately after the surgeries $(8.2 \pm 7.9 \mathrm{mmHg})$. These values remained below the threshold that would have indicated the development of a compartment syndrome.
The elevated pressure decreased over the next 5 days with the lowest value measured on day $5(1.3 \pm 1.5$ mmHg).

Evaluation of the serum levels of CK and LDH yielded a peak at day 1 after trauma and surgery, with $1035.8 \pm$ 503.3 U/L and $627.9 \pm 130.6 \mathrm{U} / \mathrm{L}$, respectively; these values decreased over the following 2 days to physiological serum levels (Figure 6).

\section{Discussion}

We showed, for the first time, the healing of a multifragmentary femoral fracture with a concurrent severe, but closed soft tissue injury in sheep. The analysis of compartment pressure measurements and evaluation of clinical markers for tissue breakdown showed a good recovery of the soft tissue injury within 5 days after trauma. Radiographic and biomechanical assessment demonstrated timely healing of the multi-fragmentary fracture within the 8 weeks experimentation time.

The combined trauma was achieved during one single

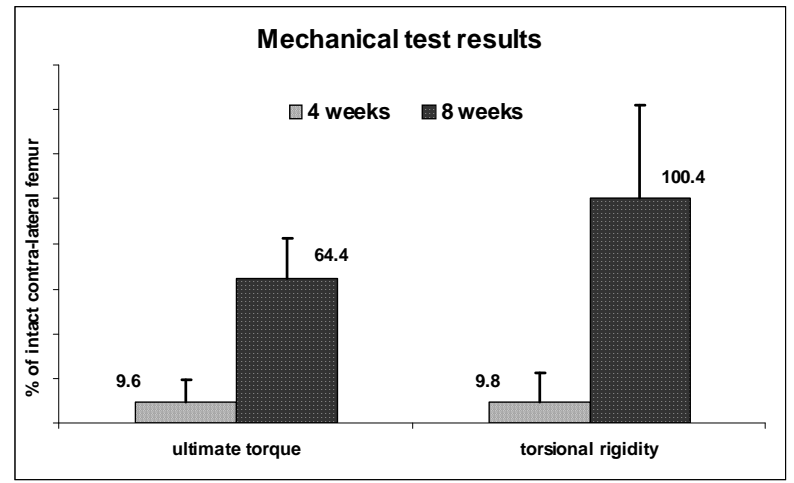

Figure 5. Mechanical testing of the two groups $(n=6)$ at 4 and 8 weeks recovery times: The ultimate torque and the torsional rigidity of the fractured femur in proportion to the uninjured contralateral femur increase significantly ( $p$ $<0.05$ ) over time.

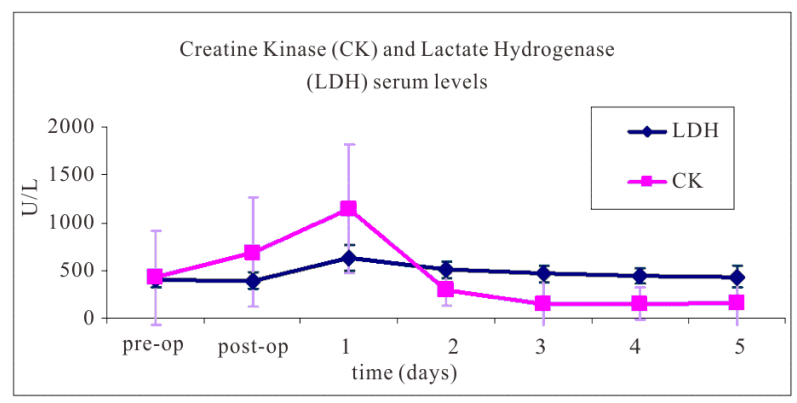

Figure 6. Average blood serum levels of Creatine Kinase and Lactate Dehydrogenase from 12 sheep over a period from just prior to the trauma application and surgery until five days after surgery. The measured values show a peak on day one after surgery, while the levels decreased to normal physiological values over the following days. 
surgery session, under general anaesthesia, within approximately 20 minutes of surgery time. The newly developed pendulum impact device created a reproducible soft tissue injury (Tscherne grade III), as demonstrated during pilot experiments by post-mortem macroscopic dissection. The AO-C3 fractures were created using procedures that could be performed through a small, trans-muscular approach, thereby minimising further iatrogenic damage to the soft tissues. The fracture pattern was consistent, enabling all fractures to be stabilised with the same configuration by using an external fixator initially, followed by an internal fixator after 5 days, resulting in a comparable mechanical environment for all animals, which further minimised the variability of the fracture healing environment.

The fracture healing was monitored with fortnightly radiographs, showing progressing callus formation with an increasing number of callus bridges, which correlated with the increase of the mechanical competency from four to eight weeks. The time course of fracture healing in our experiments corresponds well with that of previously published ovine models [9-11]. In fact, both for simple osteotomy models as well as for the multi-fragmentary fracture model by Baumgaertel, similar stiffness and strength values at comparable time points have been reported [12].

Whilst we succeeded in developing the methods to create a combined soft tissue injury and bone fracture in sheep, the chosen methods also have certain limitations. In order to have better control over the severity and reproducibility of the soft tissue injury and the multifragmentary fracture, the injuries were created in two steps. A small incision was therefore required in order to insert the instruments for the creation of the multi-fragmentary fracture. Consequently, the tissue response to the actual soft tissue injury, and to the iatrogenic injury from the fracture creation cannot be separated completely. Furthermore, this trans-muscular incision may have lead to a small fasciotomy, through which any developing compartment pressure may have been released. This could explain why only moderate compartment pressure values were measured in our experiments, and no case of compartment syndrome was observed, despite the severe muscle injury.

The recovery of the injured soft tissue was monitored by measuring the compartment pressure in the quadriceps muscle in the vicinity of the fracture zone. Peak compartment pressure values were recorded immediately post-operatively in all of the 7 sheep. Although pressure measurements were always performed whilst the animals were lying down, in order to decrease any variability due to changes in muscle tone, there was still a relatively wide distribution of the measured values for the compartment pressure. This suggests that the values were highly dependent on the positioning of the probe within the muscle, which made the measurement of absolute pressure values difficult. Nevertheless, the trend of decreasing interstitial pressure during the three days following trauma and surgery was highly consistent.

The analysis of systemic CK and LDH levels showed a peak for these diagnostic markers of tissue damage on day one after trauma/surgery, which correlates well with clinical observations of significant muscle trauma in humans. However, as the post-mortem macroscopic dissection in preliminary/pilot, terminal in vivo experiments showed, and the visual functional observation of the animals in the in vivo experiments confirmed, the injury was limited to the structure of the muscles and their surrounding soft tissues, and did not affect their function, or the structure and function of the major blood vessels and nerves. After an immediate postoperative recovery period of two hours, all sheep (with appropriate levels of analgesia) were moving their limbs without any signs of paresis. For further characterisation of the extent of the soft tissue injury and its recovery, additional experiments are planned using alternative evaluation methods, such as magnetic resonance tomography, and histological assessments.

In summary, we describe the development of a large animal model to study for the first time, the healing of a complex, reproducible fracture with concurrent closed soft tissue trauma in sheep. The results showed that the soft tissue injury recovered well and the fractures healed successfully, demonstrating the robust fracture healing in sheep. Furthermore, these results may serve as baseline data for future experiments with this clinically relevant experimental model, aimed at improving the treatment of combined soft tissue injuries and bone fractures.

\section{Acknowledgements}

This study was funded by a grant from the AO Research Foundation (W17-05), Switzerland. The implants used in this study were provided by Synthes, Switzerland. These sponsors did not have any involvement in the conception and design of the study, or acquisition of data, or analysis and interpretation of data nor any input towards this submitted article.

The authors wish to thank Peter Toggwiler and Simon Pearce (AO Institute Davos, Switzerland) for their help with the development of the experimental procedures. Prof. Mark Pearcy, Dr. Beat Schmutz, Greg Tevelen, Kimble Dunster (QUT, Brisbane, Australia) and Stan Redmond (The Prince Charles Hospital, Brisbane, Australia) for technical support, and Dr. Kathy Wilson, Dr. Sadahiro Sugiyama, Dr. Adam Freeman, William Sommers, Michael Lindeberg and Peter Lindeberg for assistance with the animal surgeries. 


\section{REFERENCES}

[1] M. Bhandari, P. Tornetta, S. Sprague, S. Najibi, B. Petrisor, L. Griffith and G. H. Guyatt, "Predictors of Reoperation Following Operative Management of fracTures of the Tibial Shaft," Journal of Orthopaedic Trauma, Vol. 17, No. 5, 2003, pp. 3-361.

[2] S. E. Utvag, O. Grundnes, D. B. Rindal and O. Reikeras, "Influence of Extensive Muscle Injury on Fracture Healing in Rat Tibia,” Journal of Orthopaedic Trauma, Vol. 17, No. 6, 2003, pp. 430-435. doi:10.1097/00005131-200307000-00007

[3] L. Claes, N. Maurer-Klein, T. Henke, H. Gerngross, M. Melnyk and P. Augat, "Moderate Soft Tissue Trauma Delays New Bone Formation Only in the Early Phase of Fracture Healing," Journal of Orthopaedic Research, Vol. 24, No. 6, 2006, pp. 1178-1185. doi:10.1002/jor.20173

[4] S. P. Bruder, D. J. Fink and A. I. Caplan, "Mesenchymal Stem Cells in Bone Development, Bone Repair, and Skeletal Regeneration Therapy,” Journal of Cellular Biochemistry, Vol. 56, No. 3, 1994, pp. 283-294. doi:10.1002/jcb.240560303

[5] R. Bielby, E. Jones and D. McGonagle, "The Role of Mesenchymal Stem Cells in maIntenance and Repair of Bone,” Injury, Vol. 38, No. Suppl 1, 2007, pp. S26-S32. doi:10.1016/j.injury.2007.02.007

[6] A. Schmeling, M. Schuetz, M. Kääb, S. Rupp, K. Schaser and K. Ito, "A Realistic Experimental Model of Shaft Fractures and Concomitant Soft Tissue Trauma in the Sheep Tibia (Abstract),” ORS 46th Annual Meeting, Or- lando, 12-15 March 2000, p. 276.

[7] H. Tscherne and H. J. Oestern, "A New Classification of Soft-Tissue Damage in Open and Closed Fractures," Unfall-Heilkunde, Vol. 85, No. 3, 1982, pp. 111-115.

[8] M. E. Mueller, S. Nazarian and P. Koch, “The Comprehensive Classification of Fractures of Long Bones,” SpringerVerlag, Berlin, Heidelberg, New York, 1990. doi:10.1007/978-3-642-61261-9

[9] R. Hente, J. Cordey, B. A. Rahn, M. Maghsudi, S. von Gumppenberg and S. M. Perren, "Fracture Healing of the Sheep Tibia Treated Using a uniLateral External Fixator. Comparison of Static and Dynamic Fixation,” Injury, Vol. 30, No. Suppl 1, 1999, pp. A44-A51. doi:10.1016/S0020-1383(99)00126-6

[10] H. Schell, H, M. S. Thompson, H. J. Bail, J. E. Hoffmann, A. Schill, G. N. Duda and J. Lienau, "Mechanical Induction of Critically Delayed Bone Healing in Sheep: Radiological and Biomechanical Results," Journal of Biomechanics, Vol. 41, No. 14, 2008, pp. 3066-3072. doi:10.1016/j.jbiomech.2008.06.038

[11] F. Baumgaertel, S. M. Perren and B. Rahn, “Animal Experiment Studies of 'Biological' Plate Osteosynthesis of Multi-Fragment Fractures of the Femur," Unfallchirurg, Vol. 97, No. 1, 2001, pp. 19-27.

[12] A. S. Malik, O. Boyko, N. Atkar and W. F. Young, “A Comparative Study of MR Imaging Profile of Titanium Pedicle Screws,” Acta Radiologica, Vol. 42, No. 3, 2001, pp. 291-293. doi:10.1080/028418501127346846 